\title{
Disseminating research results to research participants and their communities
}

\author{
Joseph Mfutso-Bengo ${ }^{1}$, Paul Ndebele ${ }^{1}$, Francis Masiye ${ }^{1}$ \\ 1. Centre for Bioethics in Eastern and Southern Africa, College of \\ Medicine, Malawi \\ Corresponding author: Professor J. M. Mfutso-Bengo, Centre for Bioethics \\ in Eastern and Southern Africa (CEBESA), Department of Community \\ Health, College of Medicine, Private Bag 360, Chichiri, Blantyre 3, \\ Malawi \\ Phone: +2651871911 ext 310, Email: mfutsobengo@medcol.mw
}

\section{Introduction}

There is wide acknowledgement of the need for community engagement in biomedical research, particularly in developing countries. Today, engaging communities has become a critical aspect of planning and implementing biomedical research. The current international research ethics guidelines talk of community engagement as an ethical requirement for research involving human subjects, particularly marginalized populations. ${ }^{1}$ It is believed that genuine community engagement offers the hope of enhancing recruitment, retention, and participant satisfaction..$^{2-7}$ However, there is relatively little published experience of community engagement in practice. Recently, the Centre for Bioethics in Eastern and Southern Africa organized dissemination workshops of results of the Wellcome Trust Bioethics Research Project as part of community engagement. The workshops were organized between the 10th of March and the 17th of April, 2008 in Madziabango, Mpemba, and Bangwe in Blantyre, and Tidziwe Centre (UNC) in Lilongwe. The workshops were attended by 32 health workers/research staff and 128 research participants including community leaders.

During the dissemination workshops, participants complained that most researchers do not engage communities in which they conduct research. Yet they feel it is very necessary for researchers to sensitize people in the communities about their research projects before initiating the projects in the communities. They said community engagement would enable community members to be aware of any research project before it is initiated in their communities and this would dispel out rumors and misconceptions that are associated with biomedical research among people. They also felt it would help researchers to work freely in the communities because people would know them and their research in advance. It could also encourage community members to enroll in biomedical research because they would be informed about the research in advance. In this paper, we analyze the issues that came out during the dissemination workshops.

\section{Objectives of the dissemination workshops}

The workshops were organized with three broad objectives;

1. To disseminate Phases 1 and 2 results of the Wellcome Trust Bioethics Research Project to research participants and research staff in Madziabango, Mpemba, Bangwe and Tidziwe Centre.
2. To discuss research results with research participants and health/research staff of the three health centers and the UNC.

3. To encourage further discussion on major challenges in conducting biomedical research in areas with limited resources.

\section{Analysis of the dissemination workshops}

After each workshop, the Bioethics Team debriefed on the proceedings of the dissemination. The team noted that the dissemination workshops were a good experience. Almost all research participants and research staff remembered the Bioethics Project and it was a wonderful reunion with the research participants and research staff. It was also noted that people participated actively in the workshops; they made useful comments and asked questions. Research participants and research staff also commended the Bioethics Team for remembering them and being exemplary in disseminating their research results. During the debriefing meetings, the Bioethics Team analyzed issues that emerged during the discussions of the disseminated results. Below are the issues that came out of the workshops;

\section{Social responsibility of researchers}

Participants noted that most of the researchers who conduct biomedical research in their communities do not care about social problems that are faced by the communities. They complained that researchers exploit them by recruiting them in their research studies without improving their lives. On this note, they asked researchers to provide some social services that are required in the communities where they conduct research in their communities. For example, one participant said, "They can show their gratitude to us by digging boreholes in the areas where water is a problem. By looking at the borehole, people will remember the research always and appreciate what the researchers did." Other participants also felt digging boreholes in communities where water is a problem would show researchers' appreciation of people's participation in their study.

Participants were also concerned about researchers who conduct hospital-based research and do not care about problems faced by health centers/hospitals where they do research. For instance, one health worker was concerned about a research institution which is conducting research at one of the health centres. She observed that the research institution has a lot of drugs (pain killers and antibiotics: Panadol and Amoxylin) which the research institution provides to research participants. However, in most cases the drugs are in large quantities and they end up expiring and are thrown away by the research staff and yet the same drugs are not available at the Health Centre and the Centre is in dire need of such drugs. She felt this was very unfair and called on researchers to be very responsive to the needs of the Health Centres/Hospitals where they conduct research. In her own words, the participant said, "What some of these 
researchers do is very unfair. They have a lot of drugs such as pain killers and antibiotics and the drugs expire right here and they throw them away. Yet at the health centre those drugs are not available and we have many patients that need them. They are aware of this but they are only concerned about their patients. I feel this is very unfair and inbuman."

Participants also noted that Clinical Officers and Nurses who work for Research Projects are usually very adequate and not very busy - however when they are asked to assist in the Hospital where their Research Clinic is, they refuse to give a hand despite knowing that the Hospital is understaffed.

Some of the workshop participants also requested that whenever they participate in biomedical research - they would like to be given a souvenir of some kind to remind them that they once participated in research. For example, they said researchers can design T-Shirts with the name of the research project which they can distribute to participants as a keepsake.

Finally, some participants felt that research participants should agree with researchers on their social responsibility and capacity building in the communities before they conduct their research.

\section{Community sensitization}

Participants complained that some researchers do not come for community sensitization before they conduct research in the communities. Instead they consult chiefs and then start doing their research. They felt this was not welcome and called on researchers to sensitize people in the communities where they would like conduct research. They stated that it is very necessary for people to be aware of any research project before it is initiated in their communities. They stated that this would dispel out rumors and misconceptions that are associated with biomedical research. They also felt that it would help researchers to work freely in the communities because people would know them and their research in advance. It could also encourage people to enroll in research because they would be informed about the research in advance. Thus one participant said, "Some researchers bribe chiefs. They come and meet them in private and convince them that they should allow their research in the community. We believe the chiefs sell us to the researchers because we are not consulted in the whole process. How do they expect people to take part in a research which has not been publicized in the communities?"

Another participant expressed disappointment at the way chiefs conduct themselves when they are approached by researchers to do research in the areas under their jurisdiction. He said, "These chiefs do not care about us. They just accept any research that comes to them even if it involves sucking blood. I am sure the researchers bribe these chiefs so that they accept their research to be conducted in the community. We are not happy with this!"

They asked the Bioethics Team to explain to researchers why there is need to sensitize people in the communities before they initiate research projects. They spoke of the need to engage communities before conducting biomedical research in the community. They felt people should be engaged because they are the ones who are asked to take part in research. On this note, some health workers complained that some research institutions do not go to communities to sensitize them about the research projects they conduct at Health
Centres. As such people are taken by surprise when they are asked to take part in research at the Health Centres. They also called on researchers to do community sensitization in the catchment areas of the health centers where they intend to conduct their research.

\section{Access to medical treatment}

Participants noted that most participants choose to participate in research out of desperation. And due to this factor, they don't pay much attention to the consent procedures. This was observed among participants in the BAN study at the UNC Project in Lilongwe. It was noted that most of the mothers recruited into the BAN study were very desperate to receive antiretroviral drugs and other drugs that were being given to participants - and due to this desperation, most of them would not pay much attention to the consent procedures. As a solution, participants suggested that after going through consent procedures, research participants should be given a comprehension test of the disclosed information. They also recommended that the consent process should be ongoing throughout the research.

\section{Dissemination of research results}

Participants requested that it should become a norm for researchers to disseminate research results to participants after the research is over. They said it is their right to know the results and this should be done promptly. In cases where the research takes long to complete, they requested that research results should be disseminated in phases and not wait for the study to finish. They said research results should not take a long time to be disseminated.

Research Staff at the UNC Project also appreciated the need to give a feedback of research results to research participants. They felt it was indeed necessary to disseminate results to research participants after completion of a study. However, they observed that it was sometimes difficult to trace research participants after the study is over because some studies take a long time to complete and by the time the study is completed, the participants might have relocated to other townships. They also noted that some results might not be of immediate benefit to research participants. All in all, it was agreed that research participants have the right to know the results of a study they participate in.

\section{Conclusion}

To conclude, we feel that the workshop participants raised very important issues that need to be addressed. We feel researchers need to adopt innovative methods of teaching communities and individuals about clinical research. We feel it is very important for researchers to engage the public before initiating any clinical research in communities - they need to sensitize the public about the research project. This engagement should also extend to communities that are used in hospital-based research. This would dispel false rumors that circulate about clinical research. It would also encourage people to participate in clinical research. This would also make people decide to participate in clinical research based on adequate information which is passed in a way that makes it easy for the public to understand. This would ensure that people make their decisions based on adequate information. We also feel researchers have social obligations to provide 
services to communities where they recruit participants in order to improve people's lives and as a way of showing gratitude to the communities. They should not use communities for their own benefit for this would tantamount to exploitation.

Research should aim at building capacity and supplementing the national health delivery system. As such, researchers should feel responsible for the needs of the institutions where they conduct research by, for example, providing personnel and supporting clinics that are open to all members of the communities and not restricted to the patients or even disease under study. They can supplement the national health delivery system by donating necessary equipment/materials to the hospitals or health centres where they have research sites. We believe that clinical research presents one route to accessing health care.

Finally, enlightened by the outcome of the dissemination workshops, the Bioethics team in the Centre for Bioethics has developed four benchmarks which could be used when engaging community members in biomedical research. The benchmarks include;

i. Community consultation/permission: Community consultation involves briefing community leaders, that is, chiefs, political leaders and religious leaders about the research project and obtaining their permission to conduct a research project in their community.

ii. Community sensitization: Community sensitization is the process of engaging community members in research. It involves explaining the research project to potential participants before they are approached to participate in research. This is done by organizing sensitization workshops/meetings in which community members are invited and informed about a research project to be initiated in the community or the local health centre/hospital.

iii. Community involvement/ownership: Community involvement is a process in which researchers ensure that community members are involved in the whole conduct of the research project and are considered as partners. It is very necessary for local communities participating in research / or providing leads to scientific findings to be considered as partners in research. This partnership should begin before the conduct of the research project and continue during the conduct of the research and after the life span of the research project. If community members are involved in the research project, they commit themselves to the activities of the project and feel ownership of the research project initiated in their community. iv Community Feedback/ dissemination of research results to participating communities: Community feedback is the dissemination of research results to research participants and their community. Researchers organize dissemination meetings to feedback the results of their research to participating community members.

The benchmarks are intended to strengthen mutual understanding between community members and research institutions. Through the complex social interactions inherent in the community engagement benchmarks, the Centre for Bioethics aims to build context specific ethical relations with local people and to strengthen understanding of how ethical principles can be applied in practice. Evaluations over time will assess the effectiveness and sustainability of these benchmarks, provide generalizable information for similar research settings, and contribute to debates on the universality of ethical principles for research.

\section{Acknowledgements}

The corresponding author is greatly thankful to the following;

- The Wellcome Trust for funding the Bioethics Research Project

- Management, Staff and research participants from: The BAN, HPTN 035 and AACTG 5175 Clinical Trials at Bwaila Hospital and Tidziwe Centre of the UNC Project in Lilongwe.

GLAM Study at Queen Elizabeth Central Hospital in Blantyre.

The Nutrition Study at the ART Clinic based at Queen Elizabeth Central Hospital in Blantyre.

\section{References}

1. Nuffield Council on Bioethics (NCB). Annual Report 2000; London: Nuffield Council on Bioethics; 2001.

2. Sapienza JN, Corbie-Smith G, Keim S, Fleischman AR. Community Engagement in epidemiological research. Ambul Pediatr.; 2007: 7 (3): $247-252$.

3. Ethical Principles and Guidelines for the Protection of Human Subjects of Research: The Belmont Report. 1979.

4. National Bioethics Advisory Commission. Ethical and Policy Issues in international research: Clinical trials in developing countries; Volume II. Bethesda. MD.

5. International Ethical Guidelines for Biomedical Research Involving Human Subjects; Council for International Organizations of Medical Sciences (CIOMS) in collaboration with the World Health Organizations (WHO). Revised Draft. Geneva: 2002.

6. Tindana PO, Singh JA, Tracy CS, Upshur REG, Daar AS, et al. (2007). Grand Challenges in Global Health: Community Engagement in Research in Developing Countries. Plos Med 2007: 4(9): e273 dio:10.1371/journal.pmed.0040273.

7. Dickert N, Sugarman J. Ethical goals of community consultation in research. Am J Public Health 2005: 95:1123 - 1127. 